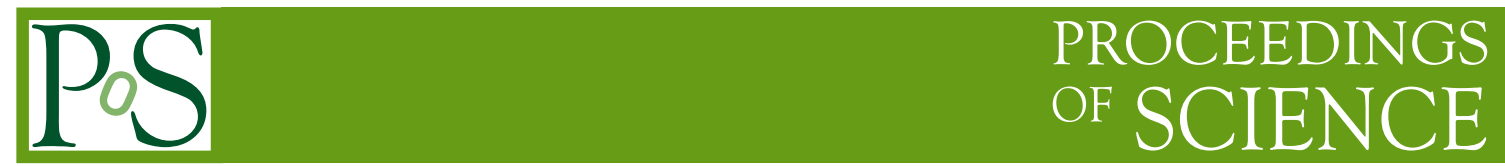

\title{
Fluctuating-Accretion Model for AGN and BHXRB X-ray Variability
}

\author{
Patricia Arévalo* \\ University of Southampton \\ E-mail: patricialastro.soton.ac.uk
}

Propagating fluctuation models can reproduce fundamental properties of the variability observed in the X-ray light curves of accreting black hole systems. We explore this type of model and show how extended emitting regions introduce at the same time energy dependent power spectral densities (PSD) and time lags between different energy bands.

VI Microquasar Workshop: Microquasars and Beyond

September 18-22 2006

Società del Casino, Como, Italy

\footnotetext{
* Speaker.
} 


\section{Propagating-fluctuations model}

\section{Accretion rate fluctuations propagating inward through the accretion flow can produce the observed wide range of variability time-scales and rms-flux relation}

$\mathrm{X}$-ray light curves from AGN and XRBs show fluctuations on a very broad range of timescales, which is hard to reconcile with the compact nature expected for the X-ray emitting region. A solution to this problem was proposed by Lyubarskii (1997)[3], where fluctuations in the accretion rate produced at different radii in the accretion flow propagate inward, to finally modulate the emission at the central, X-ray emitting region. Assuming that the characteristic variability timescales are a function of radius, e.g. proportional to the local viscous time-scale, a broad range of radii in the accretion flow can provide the broad range of variability time-scales observed. This scenario also gives a natural explanation to another important characteristic of the variability, the linear relation between the amplitude of the fluctuations and the average flux level [6]. This rmsflux relation is observed in X-ray light curves of AGN and of XRBs, regardless of whether the compact object is a black hole or neutron star, indicating that the origin of the variability lies in the accretion flow and not in the emitting mechanism. In the propagating-fluctuation model of Lyubarskii (1997), the local accretion rate fluctuations are modulated by the accretion rate fed from outer radii, so the fluctuations produced at each annulus in the accretion flow effectively multiply together, producing an rms-flux relation.
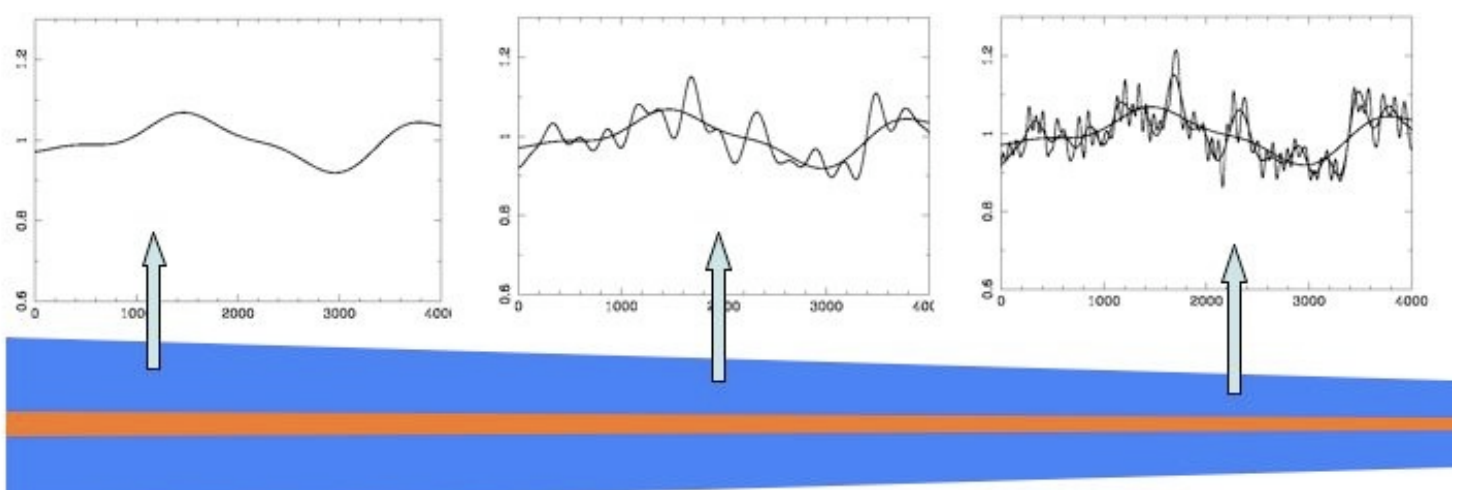

Figure 1: In this model, the accretion flow produces fluctuations in the accretion rate on the local viscous time-scale. These fluctuations propagate with the flow and modulate the accretion rate further in. Given that inner regions (towards the right in this plot) have shorter characteristic time-scales, the high frequency fluctuations get modulated by the low frequency fluctuations produced further out. This multiplicative process produces larger amplitudes of variability when the average flux is high. The plots show the local accretion rate fluctuations at three different radii, an observed light curve can be the sum of variability patterns from several different radii, whose contributions to the total flux are determined by the radial emissivity profile.

As noted by Churazov et al. (2001)[2], the high amplitude, high frequency variability of the power law component in the spectrum of XRBs indicates that it originates in a thick accretion flow, where the short characteristic time-scales allow fluctuations in the accretion rate to propagated without being significantly damped, and high frequency variability to be produced. They show that a geometrically thick accretion flow sandwiching a thin, thermally emitting, disc can explain the strong variability of the power law and the stability of the black body components simultaneously. 

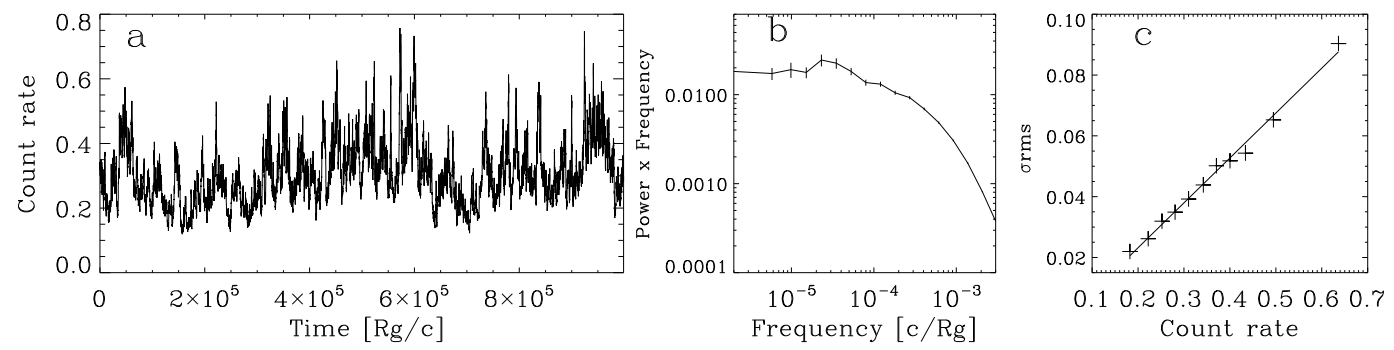

Figure 2: Simulated light curve (a), its Power spectrum (b) in terms of Power $\times f$, shows a flat top (corresponding to Power $\propto 1 / f$ ), that cuts off at high frequencies due to the filtering effect of an extended emitting region. Simulated light curves also reproduce the rms-flux relation (c).

In their model, the variability arises as accretion rate fluctuations in the thick flow or corona, which propagate inward to the main X-ray emitting region. We will explore this scenario to show that it can reproduce many characteristics or the X-ray variability.

Following Lyubarskii (1997)[3], we assumed that independent fluctuations are introduced at geometrically spaced radii, with a radially-dependent characteristic time-scale and that they propagate inward with viscous velocity (see [1] for details of the numerical implementation). Light curves produced numerically following this procedure (Fig. 2a) have a PSD $\propto 1 / f$ (Fig. 2b) with a high frequency cut-off, as observed in many AGN and XRBs in the high/soft state [4]. The method also reproduces the linear relation between rms variability and flux (Fig. 2c) seen in both types of objects ([6]).

\section{The effect of extended emitting regions}

\section{Different emissivity profiles for different energy bands produce energy-dependent power spectra and time lags.}

Observationally, softer X-ray light curves show less variability on short timescales and tend to lead the variability of harder bands. This behaviour can be achieved by the model if the energy spectrum of the emitting region hardens towards the centre, i.e. if the softer X-rays are emitted by a more extended region. Figure 3 shows the PSDs of three simulated light curves, which differ only by the extent of their emitting regions. The solid line represents the PSD of a light curve emitted only at the innermost radius, the other two PSDs demonstrate the effect of extended emitting regions applied on the same variability pattern: the dotted line corresponds to a radial emissivity profile $\varepsilon(r) \propto r^{-3}$ and the dashed line corresponds to $\varepsilon(r) \propto r^{-5}$. The flatter the emissivity profile, the more high-frequency variability power is suppressed. Also, as the fluctuations propagate towards the central black hole, they are seen first in the more extended emitting region (soft) and later in the more concentrated emitting region (hard), which produces hard time-lags. These lags increase both with the time-scale of the fluctuations and with the difference in emissivity indices (see Fig. 4). The lag spectrum calculated between the simulated light curves that produce the PSDs in Fig. 3 is shown in Fig. 4. 


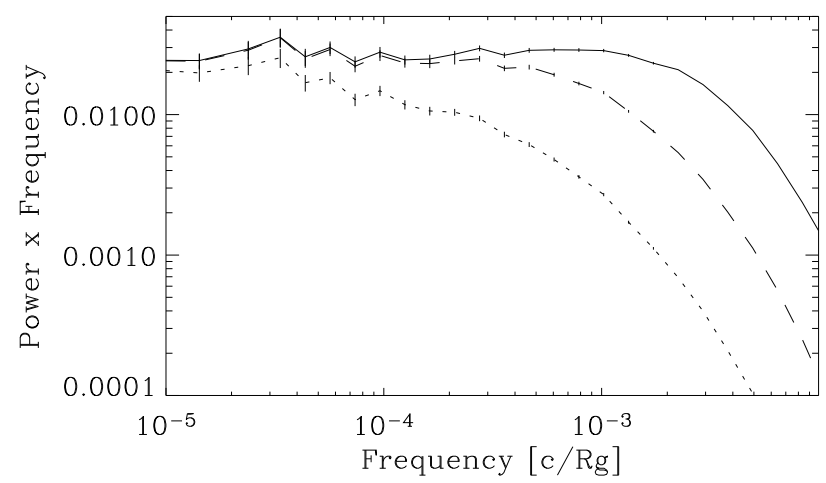

Figure 3: Effect of an extended emitting region: PSDs of three simulated light curves produced from the same variability pattern in the accretion flow, the solid line represents the PSD emitted only at the innermost radius while the other two have contributions from a range of radii. The PSD shown by the dashed line has been folded through an extended emitting region with emissivity $\varepsilon(r) \propto r^{-5}$ per unit area, while the dotted line has $\varepsilon(r) \propto r^{-3}$. The more extended the emitting region, the more high-frequency power is suppressed.

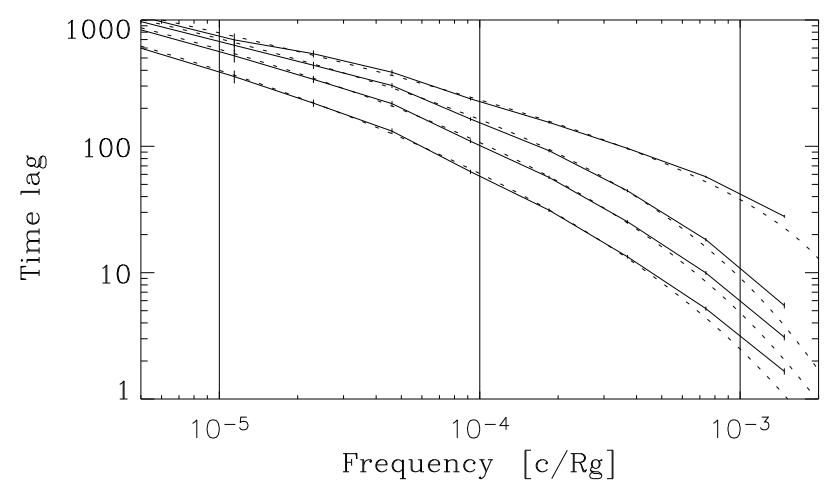

Figure 4: Lag spectra of simulated light curves. The light curves in different "bands" are produced by folding the same variability pattern through different emitting regions, characterised by the steepness of their emissivity profiles, $\varepsilon(r) \propto r^{-\gamma}$. For this plot the "soft" band has $\gamma=3$ and the "hard" bands have values of $\gamma$ of 3.5, 4, 5 and $\infty$. The fact that, in the model, the fluctuations propagate inward and harder energy bands are more centrally concentrated produces hard lags. The resulting lag spectra have power law slopes of -0.8 to -0.5 at low frequencies and curve slowly to steeper slopes at higher frequencies. The values of the lags are around 1 to $10 \%$ of the variability time-scale and increases with the difference in emissivity indices.

\section{Fit to Cyg X-1 data in the high/soft state}

To check that the magnitude of the energy dependence of the PSD and the lags in real data can be reproduced consistently by a given pair of emissivity profiles, we compared model predictions to data of Cyg X-1 in the high/soft state. We used data in $2-5 \mathrm{keV}$ for the soft band and 8-13 keV for the hard band and computed the ratio of their PSDs, to highlight their energy dependence. This PSD ratio, together with the lag spectrum between these bands, is plotted in Fig. 5. The solid lines in this plot represent a model fit, calculated assuming an emissivity profile of $\varepsilon(r) \propto r^{-3}$ for the soft band and obtaining a profile of $\varepsilon(r) \propto r^{-3.5}$ for the hard band. This small difference in emissivity 
profiles can reproduce the weak energy dependence of the PSDs and yet produce a lag spectrum of the right amplitude and frequency dependence. We assumed thick accretion flow parameters to relate the variability time-scales to radial positions, the propagation velocity obtained from this fit is approximately half of the corresponding viscous velocity, showing that if the characteristic variability time-scales are similar to the propagation time-scales (e.g. both proportional to the viscous time-scale), then time lags of the right amplitude are produced.

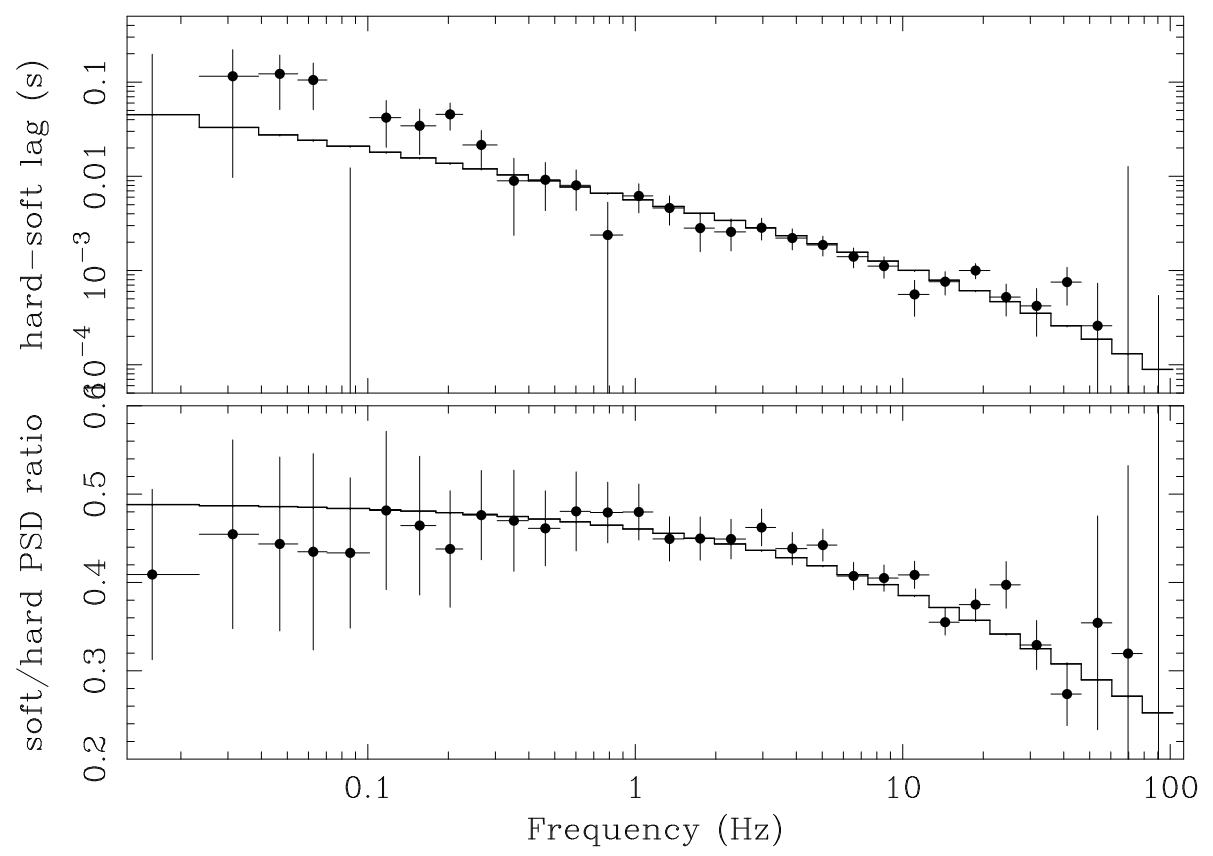

Figure 5: Cyg X-1 high state data: the top panel shows the lag spectrum between $2-5 \mathrm{keV}$ and $8-13$ $\mathrm{keV}$ energy bands and the bottom panel shows the ratio between the PSDs of both bands. The hard band lags the soft band and shows relatively more high-frequency variability power. The solid lines on both panels represent a model fit to these data. The amplitude of the lags and general shape of the spectra could be reproduced fairly well assuming simple power laws for the emissivity profiles of both bands and a propagation velocity of the fluctuations of approximately half the viscous velocity of a standard thick disc.

\section{PSD peaks and lag spectrum steps}

Lags produced by propagating fluctuation through extended emitting regions provide a natural explanation for the connection between peaks in the PSD and steps in the lag spectrum, seen for example in Cyg X-1 in the low/hard state [5]. In this scenario the value of the time lags is determined by the radius where the fluctuations originate (together with the different emissivity profiles for each energy band), so all variability time-scales produced at a given radius will have the same time-lag. PSDs composed of a few lorentzian components can be produced by the propagating fluctuation model if only a few radii contribute to the variability. The effect of the extended emitting region then is to create a stepped lag spectrum (Fig. 6), where the steps go from the time lag value associated with one of these radii to the lag of the next. 


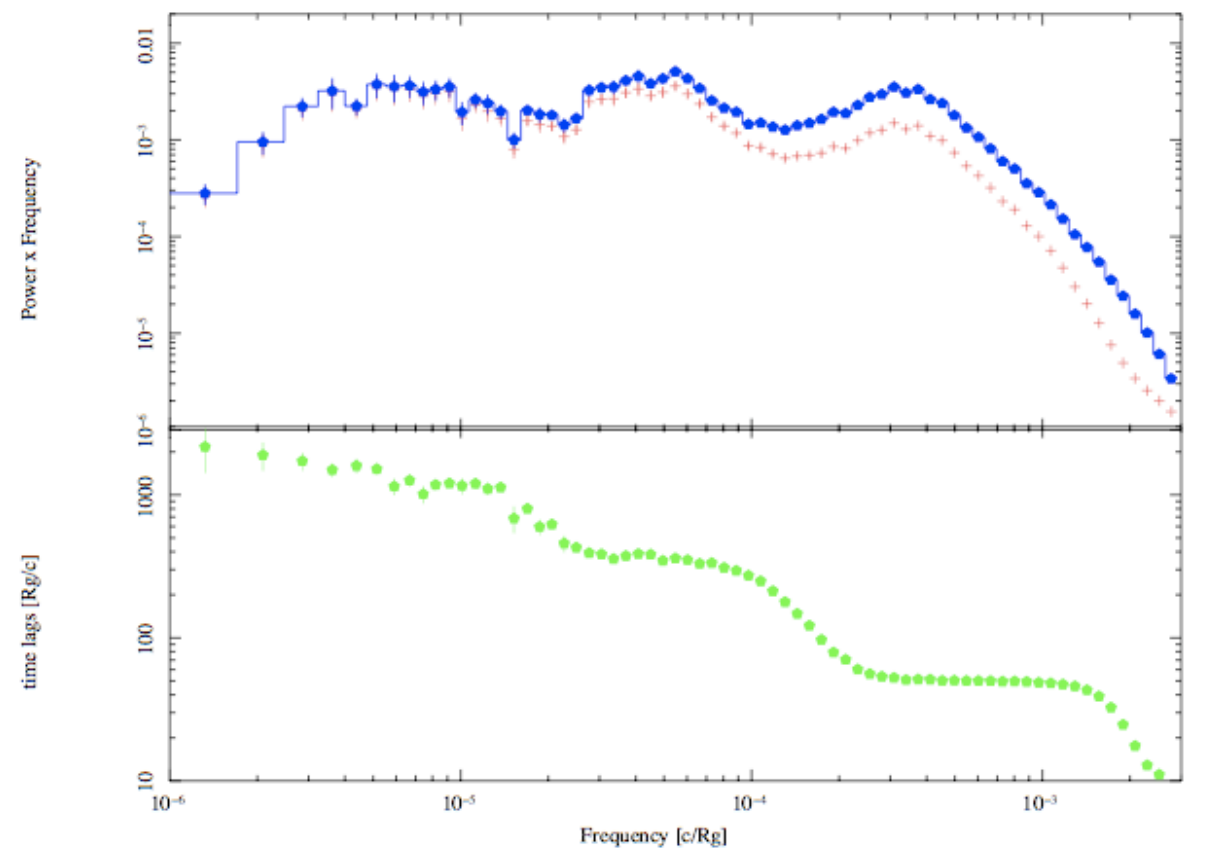

Figure 6: PSDs (top) and lag spectrum (bottom) of two simulated light curves produced by assuming that only three annuli in the accretion flow contribute to the variability. Each varying annulus produces a Lorentzian profile PSD peaking at the local viscous time-scale. The light curves are calculated with different emissivity profiles which produces the difference in the high frequency power and the time lags. Assuming that the emitted spectrum hardens towards the centre, the PSD in blue dots would represent the hard band and the red crosses the soft band, and positive values in the lag spectrum would represent hard lags. As in this model the value of the lags is associated with the radius of origin of the variability, all the frequencies dominated by a single component (lorentzian) will have a single lag. The resulting lag spectrum is therefore composed of steps, constant values where one lorentizan clearly dominates the variability and sharp drops between these values at frequencies where two lorentzians overlap. The amplitude of the lag spectrum is mainly determined by the propagation velocity of the fluctuations, in this case this velocity was assumed to be equal to the viscous velocity in a thick accretion disc.

\section{Conclusions}

The general propagating fluctuation model can reproduce several observed variability properties of the X-ray light curves of accreting object. In its simplest realisation, these include the broad range of variability time-scales and the rms-flux linear relation. Complemented with an extended emitting region, possibly and extended corona interior to or overlying a thin disc, with an energy spectrum that hardens toward the centre, this model also reproduces the energy dependence of the PSDs and the hard-lags, observed in many AGN and XRBs. This mechanism to produce time-lags does not require any spectral evolution of the emitting region, and naturally relates the peaks in the PSD to steps in the lag spectrum, observed in cases where the PSD is composed of a few different lorentzian components. 


\section{References}

[1] Arévalo, P., Uttley, P. 2006, MNRAS 367, 801

[2] Churazov, E., Gilfanov, M., Revnivtsev, M. 2001, MNRAS 321, 759

[3] Lyubarskii, Y. 1997, MNRAS 292, 679

[4] McHardy I. M., Gunn K. F., Uttley P., Goad M. R. 2005, MNRAS 359, 1469

[5] Nowak, M. A. 2000, MNRAS 318, 361

[6] Uttley, P., McHardy I. 2001, MNRAS 323, 26 